\title{
ABUNDÂNCIA E SAZONALIDADE DE DÍPTEROS (INSECTA) EM GRANJA AVIÁRIA DA REGIÃO NORDESTE DO ESTADO DE SÃO PAULO, BRASIL*
}

\author{
WELBER DANIEL Z. LOPES ${ }^{1}$; FÁBIO H. DA COSTA²; WILTON CARLOS Z. LOPES ${ }^{3}$; JÚLIO CÉSAR \\ DE C. BALIEIRO ${ }^{4}$; VANDO E. SOARES ${ }^{5}$; ÂNGELO P. DO PRADO ${ }^{5}$
}

\begin{abstract}
LOPES, W.D.Z.; COSTA, F.H. DA; LOPES, W.C.Z.; BALIEIRO, J. C. DE C.; SOARES, V.E.; PRADO, A.P. DO. [Abundance and seasonality of Diptera (Insecta) in a poultry house in the northeast region of the state of São Paulo, Brazil]. Abundância e sazonalidade de dípteros (Insecta) em granja aviária da região nordeste do estado de São Paulo, Brasil. Revista Brasileira de Parasitologia Veterinária, v. 17, n. 1, p. 21-27, 2008. Departamento de Medicina Veterinária Preventiva, Faculdade de Ciências Agrárias e Veterinárias/CPPAR. Universidade Estadual Paulista "Júlio de Mesquita Filho", Via de acesso prof. Paulo Donatto Castellani, s/n Jaboticabal, SP. CEP.:14884-900. E-mail: wdzlopes@fcav.unesp.br

Modern production systems accommodate broody hens in high densities, leading to the accumulation of excrement under the cages. This substrate is excellent for the development of sinantropic flies. Thus, the accomplishment of surveys in these places becomes essential, in order to plan better strategies of control. The present work aimed at studying the entomofauna and the seasonality of the species of dipterous present in the Crisdan poultry house located in the Municipality of São João da Boa Vista, the State of São Paulo, Brazil. In the period of January of 2001 to December of 2002, 1,012,595 flies were captured using the "jug-trap". The species were identified: Drosophi-la repleta (Wollaston, 1858), Musca domestica (Linnaeus, 1758), Ophyra spp., Hermetria illucens (Linnaeus, 1758), Fannia canicularis (Linnaeus, 1761), Chrysomya megacephala (Fabricius, 1794), and Sepsidae. More frequently D. repleta and $M$. domestica had added $99.47 \%$ of the dipterous. Increased rainfall and the collection months influenced the sampling of dipterous $(\mathrm{P}<0.05)$. Drosophila repleta was the most abundant species, representing $91 \%$ of all captured flies. However, this diptera did not develop at the surveyed site since immatures were not captured therein.
\end{abstract}

KEY WORDS: Arthropod; flies; Drosophila repleta; poultry house.

\section{RESUMO}

Nos sistemas modernos de produção de ovos, as aves poedeiras são alojadas em altas densidades, acumulando esterco sob as gaiolas. Esse substrato é excelente para o desenvolvimento de moscas sinantrópicas. Sendo assim, se torna essencial a realização de levantamentos de moscas nesses locais, a fim de se

\footnotetext{
* Sob os auspícios do Centro Universitário UNIFEOB.

${ }^{1}$ Departamento de Medicina Veterinária Preventiva, Faculdade de Ciências Agrárias e Veterinária FCAV/UNESP/CPPAR, Via de acesso prof. Paulo Donatto Castellani, s/n Jaboticabal, SP. CEP.:14884-900. E-mail: wdzlopes@fcav.unesp.br

${ }^{2}$ Médico Veterinário autônomo.

${ }^{3}$ Departamento de Biologia Celular e Molecular e Bioagentes Patogênicos, Faculdade de Medicina, Ribeirão Preto, Universidade de São Paulo (USP). Ribeirão Preto, SP.

${ }^{4}$ Departamento de Ciências Básicas. Faculdade de Zootecnia e Engenharia de Alimentos de Pirassununga, USP. Pirassununga, SP.

${ }^{5}$ Universidade Camilo Castelo Branco. Descalvado, SP.

${ }^{6}$ Departamento de Parasitologia, Instituto de Biologia, Universidade Estadual de Campinas. Campinas, SP.
}

planejar uma melhor estratégia de controle. O presente trabalho teve como objetivo estudar a entomofauna e a sazonalidade das espécies de dípteros da granja Crisdan, em São João da Boa Vista, São Paulo, Brasil. No período de janeiro de 2001 a dezembro de 2002 foram capturados 1.012.595 exemplares utilizando-se armadilha "jug-trap". As espécies identificadas foram: Drosophila repleta (Wollaston, 1858), Musca domestica (Linnaeus, 1758), Ophyra spp., Hermetria illucens (Linnaeus, 1758), Fannia canicularis (Linnaeus, 1761), Chrysomya megacephala (Fabricius, 1794) e Sepsidae. Com maior freqüência $D$. repleta e $M$. domestica representaram 99,47\% dos dípteros capturados. A elevação dos índices pluviométricos em alguns meses dos anos influenciaram a amostragem de dípteros $(\mathrm{P}<0,05)$. $D$. repleta representou cerca de $91 \%$ de toda população. Entretanto, este díptero provavelmente não estava se desenvolvendo no local, já que estágios imaturos não foram capturados.

PALAVRAS-CHAVE: Artrópodes; moscas; Drosophila repleta; granja avícola. 


\section{INTRODUÇÃO}

O crescimento populacional tem impulsionado uma maior produção avícola para atender à demanda de consumo de proteínas. Em conseqüência disto, o processo de industrialização para a produção e comercialização de alimentos de origem animal torna-se uma necessidade, tentando diminuir a defasagem existente entre produção e consumo (GIANIZELLA; PRADO, 1998).

No caso da produção industrial de ovos, as criações foram se adaptando às novas exigências do mercado, aumentando o número de animais confinados por área (GUIMARÃES, 1985). De acordo com Axtell e Arends (1990), nos sistemas modernos de produção de ovos, as aves são alojadas em altas densidades, com o conseqüente acúmulo de excrementos sob as gaiolas. Esse substrato torna-se excelente para colonização e desenvolvimento de espécies de insetos sinantrópicos de grande importância médico sanitária (AXTELL, 1986; POLVONY, 1971).

Segundo North e Bell (1990), o aumento do número de moscas ocorre concomitantemente com a contínua expansão das cidades, em direção à zona rural. Uma galinha com 1,8 kg em atividade de postura produz, por dia, cerca de $113 \mathrm{~g}$ de fezes úmidas, o suficiente para sustentar pelo menos 100 larvas de Musca domestica por dia.

Bicho et al. (2004) relataram que o problema dos patógenos transmitidos pelas moscas vem aumentando drasticamente nos últimos anos, com tendência a se agravar no futuro, pois a população desses dípteros, freqüentemente é carreada para as cidades próximas das granjas avícolas durante os meses de verão, aumentando ainda mais os problemas de patógenos transmitidos por estes vetores. Ainda, segundo os mesmos autores, para que se realize um controle integrado de moscas é necessário principalmente entender a biologia e o comportamento das espécies de dípteros na área onde está o sistema criatório.

A espécie de díptero mais abundante no esterco acumulado de granjas é a $M$. domestica L. (Muscidae), devido ao fato de depender exclusivamente de substratos produzidos pelo homem (POLVONY, 1971).

Greenberg (1971) citou uma lista de mais de 50 espécies de patógenos que podem ser veiculados e transmitidos pela M. domestica. Entre eles, se encontram o vírus da Newcastle e, no caso de suas larvas se alimentarem em cadáveres, bactérias como Clostridium botulinum (Ermengem, 1897) e Mycobacterium tuberculosis (Koch, 1882) (HOFSTAD et al., 1984). Segundo Avancini e Ueta (1990) esta mesma espécie também pode ser hospedeiro intermediário de Choanotaenia infundibulum (Bolch 1779) um cestóide específico difundido em criação de galinhas.

Com intuito de obter a diminuição dessas populações de dípteros, um programa de controle deve ser elaborado com métodos culturais, biológicos e químicos (CARRARO; AZEVEDO, 2000). Para isso, torna-se essencial realizar o levantamento dessas espécies a fim de se planejar estratégias adequadas de controle dessas moscas.
O trabalho teve como objetivos realizar o levantamento das espécies de dípteros na granja aviária e verificar a sazonalidade e abundância relativa dessas espécies durante dois anos, visando um possível controle por meio de métodos integrados.

\section{MATERIAL E MÉTODOS}

\section{Descrição da Granja}

As coletas foram realizadas na granja Crisdan, situada a 7 km do município de São João da Boa Vista, $\left(22^{\circ} 01^{\prime}\right.$ S, $046^{\circ}$ 48’ $\mathrm{O}$, altitude de 763 metros).

A granja, considerada de pequeno porte, é constituída por dois conjuntos de quatro galpões abertos, e tem capacidade para alojar 25.000 galinhas das linhagens "Hy Line” e "Hyline Brown”, em fase de postura.

No galpão de coleta há três conjuntos de gaiolas, separados por dois corredores de concreto de 0,5 m de largura. As fileiras das gaiolas estão distribuídas em degrau, ou seja, uma fileira mais interna a $0,5 \mathrm{~m}$ do chão e uma mais externa a $1 \mathrm{~m}$ (tipo "narrow house"). As gaiolas variam de tamanho, podendo abrigar de 2 a 4 galinhas. Sob as gaiolas, o chão é de terra, onde as fezes se acumulam.

Ao redor da granja havia cultura agrícola e, durante o período analisado, a predominância foi de café e milho. Durante a coleta, nenhum inseticida foi utilizado sobre o esterco, e a maior parte do mesmo foi retirado em 24/04 e 20/09 de 2001 e em 15/03 e 02/11 de 2002. Devido às altas precipitações constatadas no verão, o uso de óxido de cálcio e de serragem foi relativamente comum nesta época do ano.

Foram realizadas setenta e cinco coletas entre 17/01/2001 a 26/12/2002, sendo cada coleta realizada com intervalos de sete dias durante o primeiro ano, passando a quinzenais nos dozes meses finais, o que totalizou 750 armadilhas expostas no galpão ao longo de todo o período.

\section{Armadilha Aérea (jug-trap)}

Para amostragem dos espécimes adultos, foi utilizado o método “jug-trap” (BURG; AXTELL, 1984; LYSYK; AXTELL, 1986). Tal método consiste na utilização de frascos plásticos com 17 centímetros de altura por sete centímetros de diâmetro, com quatro orifícios de três centímetros de diâmetro cada um, dispostos lateralmente. Estas armadilhas estiveram dispostas ao longo de dois corredores A e B, em dez pontos distintos aleatoriamente sorteados, a uma altura de 60 centímetros do solo e do substrato. No interior de cada armadilha foi colocado cerca de $10 \mathrm{~g}$ de uma isca atrativa sexual específica para moscas à base de: metanol $90 \%+$ muscamone (60\% Z-9-tricosene) + bitrex (GIANIZELLA; PRADO, 1998).

A distribuição das armadilhas foi feita por "amostragem sistemática”, que consistiu em delimitar áreas em quadrados contíguos $\left(1 \mathrm{~m}^{2}\right)$, onde as mesmas foram distribuídas (KREBS, 1989).

A cada coleta, as armadilhas foram retiradas e substituídas por novas contendo a mesma quantidade de isca. Todo 
material coletado foi levado para o laboratório de Entomologia Aplicada, e em seguida foi triado, contado, identificado, fixado e conservado em álcool a $70 \%$.

A identificação dos dípteros foi realizada por meio de chaves de identificação (CURRAN, 1965; BORROR; WHITE, 1970; MARICONI et al., 1999; CARVALHO; RIBEIRO, 2000). O material testemunho encontra-se na Coleção Entomológica da Faculdade de Medicina Veterinária do Centro Universitário das Faculdades Integradas da Fundação de Ensino "Octávio Bastos".

Foram verificadas as principais fontes de variação que poderiam influenciar no número de moscas (por gênero e/ou espécie), avaliando-se as significâncias dos efeitos de coleta, local, mês e ano, além dos efeitos de temperatura, umidade relativa do ar e precipitação pluviométrica incluídas como covariáveis. Nas análises estatísticas, adotou-se a transformação de $\log (x+1)$ ou escala raiz quadrada no número total de insetos capturados mais um (por gênero e/ou espécie) (BANZATTO; KRONCA, 1989). As análises finais, contemplando somente as fontes de variação significativas foram descritas por meio de um modelo que considerou os efeitos de mês e ano de captura, além da covariável linear pluviosidade mensal, ou seja:

$$
\mathrm{y}_{\mathrm{ijk}}=\mu+\mathrm{M}_{\mathrm{i}}+\mathrm{A}_{\mathrm{j}}+\mathrm{b}_{1}\left(\text { Pluv }_{i j k}-\overline{P l u v}\right)+\mathrm{e}_{\mathrm{ijk}}
$$

em que: $y_{i j k}$ é o valor transformado para escala raiz quadrada do número total de dípteros capturados mais um no mês i, do ano j; $M_{i}$ é o efeito do i-ésimo mês de captura; $A_{j}$ é o efeito do j-ésimo ano de captura; $b_{1}$ é o coeficiente de regressão linear da característica $y_{i j k}$ em relação a pluviosidade; $P l u v_{i j k}$ é a pluviosiodade no ano j e no mês i incluída no modelo como covariável; $\overline{P l u v}$ é a média das precipitações pluviométricas observadas durante o estudo; eijk é o efeito aleatório residual associado às observações, no ano j e no mês $i$, assumido com média 0 e variância $\sigma_{\mathrm{e}}^{2}$.
Face à covariável precipitação pluviométrica (com efeito linear) ter apresentado resultados significativos para algumas espécies avaliadas, as análise das correlações momento-produto de Pearson foram utilizadas para avaliação da associação entre os números de dípteros capturados e os índices pluviométricos. O teste para verificação de correlações, realizado pelo programa supracitado, foi Teste $t$ de Student, específico para correlações. Para tanto, foram utilizados os procedimentos GLM do programa Statistical Analysis System, versão 6.12 (SAS, 1995).

\section{RESULTADOS E DISCUSSÃO}

Durante os 24 meses de amostragem foram coletados 1.012.595 dípteros adultos (Tabela 1), pertencentes a seis famílias: Drosophilidae, Drosophila repleta (Wollaston, 1858); Muscidae, Musca domestica (Linnaeus, 1758) e Ophyra spp.; Sepsidae; Stratiomyiidae, Hermetria illucens (Linnaeus, 1758); Fanniidae, Fannia canicularis (Linnaeus, 1761) e Calliphoridae, Chrysomya megacephala (Fabricius, 1794).

A temperatura durante o período de coleta apresentou mínima de $16^{\circ} \mathrm{C}$ (junho de 2001) e máxima de $34^{\circ} \mathrm{C}$ (janeiro e outubro de 2001), umidade relativa do ar apresentou mínima de 24\% (agosto de 2001) e máxima de 100\% (maio de 2001), para pluviosidade foi notificada mínima de $0 \mathrm{~mm}^{3}$ nos meses de julho de 2001 e 2002 e máxima de $341 \mathrm{~mm}^{3}$ em novembro de 2001 (Tabela 1).

A família Drosophilidae, representada por $D$. repleta, foi a mais abundante, correspondendo a 929.367 ou $91,78 \%$ de todos os dípteros capturados, seguida da família Muscidae, representada por $M$. domestica com 77.874 (7,69\%) e Ophyra spp. com 5.196 (0,51\%) dípteros adultos (Figuras 1, 2 e 3).

A elevada abundância de Drosophilidae difere da maioria dos estudos em granjas de aves poedeiras realizados em outros locais (BRUNO et al., 1993; GIANIZELLA, 1995;

Tabela 1. Dípteros sinantrópicos coletados por armadilhas "jug-trap" em granja avícola de São João da Boa Vista, São Paulo, Brasil e parâmetros climáticos de janeiro de 2001 a dezembro de 2002.

\begin{tabular}{|c|c|c|c|c|c|c|c|c|}
\hline Família & \multicolumn{2}{|c|}{ Gênero/Espécie } & $\mathrm{N}^{\mathrm{a}}$ & $\bar{x}$ & DP & \multicolumn{2}{|c|}{ A } & TOTAL $^{b}$ \\
\hline Drosophilidae & \multicolumn{2}{|c|}{ Drosophila repleta* } & 75 & $12.392,00$ & 48302,7 & \multicolumn{2}{|c|}{$(0-64.286)$} & $929.367,00$ \\
\hline Muscidae & \multicolumn{2}{|c|}{ Musca domestica* } & 75 & $1.038,32$ & 4401,87 & \multicolumn{2}{|c|}{$(0-12.425)$} & $77.874,00$ \\
\hline Ophyra spp. ${ }^{\text {ns }}$ & & 75 & 69,28 & 298,91 & $(0-456)$ & \multicolumn{2}{|c|}{$5.196,00$} & \\
\hline Sepsidae & & - & 75 & 1,44 & 5,9 & \multicolumn{2}{|c|}{$(0-16)$} & 108,00 \\
\hline Stratiomyiidae & Herme & tria illucens ${ }^{\text {ns }}$ & 75 & 0,26 & 1,72 & \multicolumn{2}{|c|}{$(0-15)$} & 20,00 \\
\hline Fanniidae & Fannia & canicularis ${ }^{\mathrm{ns}}$ & 75 & 0,2133 & 1,37 & \multicolumn{2}{|c|}{$(0-5)$} & 16,00 \\
\hline Calliphoridae & Chrysomy & a megacephala & 75 & 0,1867 & 2,1 & \multicolumn{2}{|c|}{$(0-10)$} & 14,00 \\
\hline \multicolumn{9}{|c|}{ Covariáveis } \\
\hline \multicolumn{3}{|c|}{ Temperatura } & 75 & 26,29 & 4,24 & 16 & 34 & - \\
\hline \multicolumn{2}{|c|}{ Umidade Relativa } & - & 75 & 66,15 & 20,29 & 24 & 100 & - \\
\hline \multicolumn{2}{|c|}{ Precipitação Pluviométrica } & - & 75 & 139,89 & 117,16 & 0 & 341 & - \\
\hline
\end{tabular}

Número de coletas $(\mathrm{N})$, média $(\bar{X})$, desvio padrão (DP), amplitude (A) e número total de indivíduos coletados (TOTAL)

* Significativo $(P<0,05)$ para índice pluvial;

** Significativo $(P<0,01)$ para índice pluvial;

ns Significativo $(P>0,05)$ para índice pluvial. 


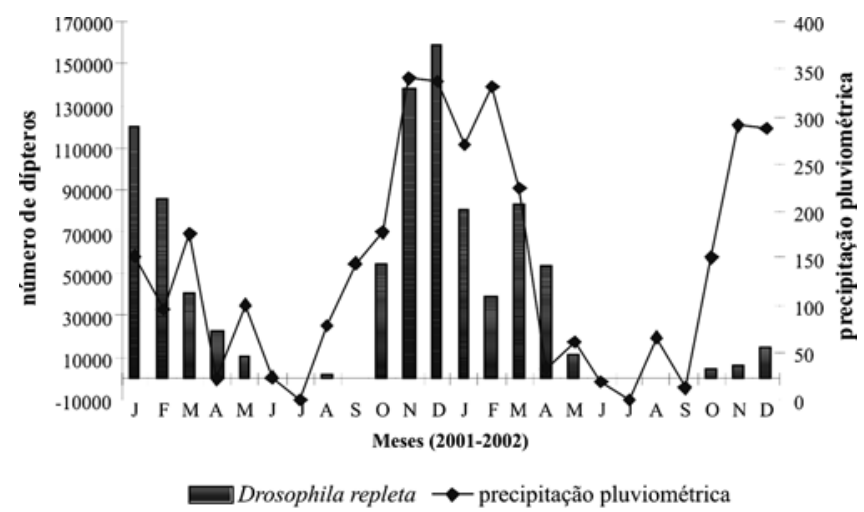

Figura 1. Dinâmica populacional de Drosophila repleta capturadas por armadilhas "jug-trap" durante os anos de 2001 e 2002, em granja avícola de São João da Boa Vista, São Paulo, Brasil.

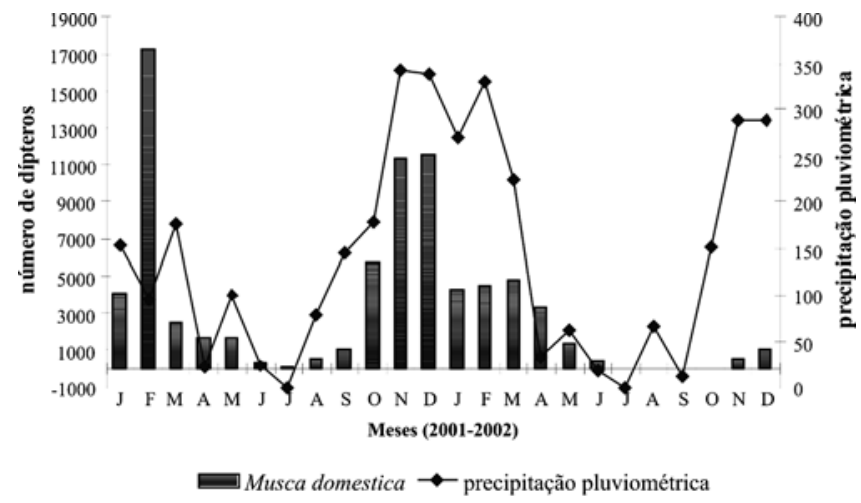

Figura 2. Dinâmica populacional de Musca domestica capturadas por armadilhas "jug-trap" durante os anos de 2001 e 2002, em granja avícola de São João da Boa Vista, São Paulo, Brasil.

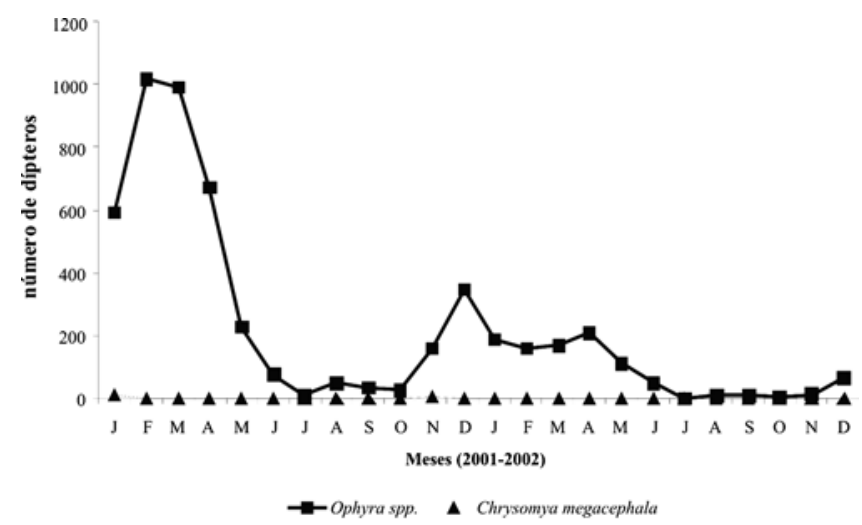

Figura 3. Dinâmica populacional de Ophyra spp. e Chrysomya megacephala capturadas por armadilhas "jug-trap" durante os anos de 2001 e 2002, em granja avícola de São João da Boa Vista, São Paulo, Brasil.

AVANCINI; SILVEIRA, 2000; BICHO et al., 2004). Vale ressaltar que Bicho et al. (2004), notificaram em Pelotas, RS, a espécie $D$. repleta em excrementos aviários como sendo a segunda mais abundante (9.229 insetos ou 19,75\%) de sua amostragem. Os demais autores constataram a família Muscidae como sendo a mais abundante.
Musca domestica foi assinalada sendo como o díptero mais abundante em levantamentos em granjas avícolas realizados em Uberlândia, MG (LOMÔNACO; PRADO, 1994) e em Monte Mor, SP (AVANCINI; SILVEIRA, 2000) representando $91,82 \%$ e $83,16 \%$ das populações amostradas respectivamente. Bruno et al. (1993), em diversas granjas do estado de São Paulo, registraram esta espécie como sendo a segunda mais abundante (30,11\% das larvas). De forma semelhante, em Uberlândia, $M$. domestica foi a terceira espécie mais abundante (FERNANDES et al., 1995). Entretanto, Bicho et al. (2004) em granja aviaria de Pelotas, RS, capturaram apenas 143 indivíduos desta espécie (0,30\%).

Olton e Legner (1970) observaram em granja avícola no sul da Califórnia a maior abundância de $M$. domestica em relação Fannia spp.

Em diversas granjas de aves poedeiras, Bruno et al. (1993) concluíram que $M$. domestica apresenta relação direta com a umidade do esterco, procriando em esterco de umidade relativa de $45 \%$ a $64 \%$, coincidindo com os dados encontrados no presente trabalho, já que a umidade média do esterco durante o período amostrado foi de $61,21 \%$. Peck e Anderson (1969) relataram que larvas de $M$. domestica predominaram em esterco de galinhas contendo acima de $70 \%$ de umidade. Miller et al. (1988) encontraram 60-75\% e Stafford e Bay (1987) 70-79\% como melhor faixa de umidade no do ar para desenvolvimento de larvas desta espécie.

Sepsidae, Stratyiiomidae (H. illucens), Fanniidae (F. canicularis) e Calliphoridae (C. megacephala) somaram 158 (0,015\%) moscas evidenciando sua baixa abundância (Figuras 3 e 4). A espécie $C$. megacephala, foi a menos abundante com apenas 14 exemplares capturados durante os 24 meses, das quais 10 foram capturadas em janeiro de 2001 (Tabela $1 \mathrm{e}$ 2). Vale ressaltar que, segundo Peck e Anderson (1970), a presença ou ausência de califorídeos em aviários decorre principalmente da existência ou não de aves mortas e ovos quebrados no galpão.

Espécies de Fannia spp. notificadas nos trabalhos realizados em granjas aviárias dos EUA por Axtell e Arends (1990);

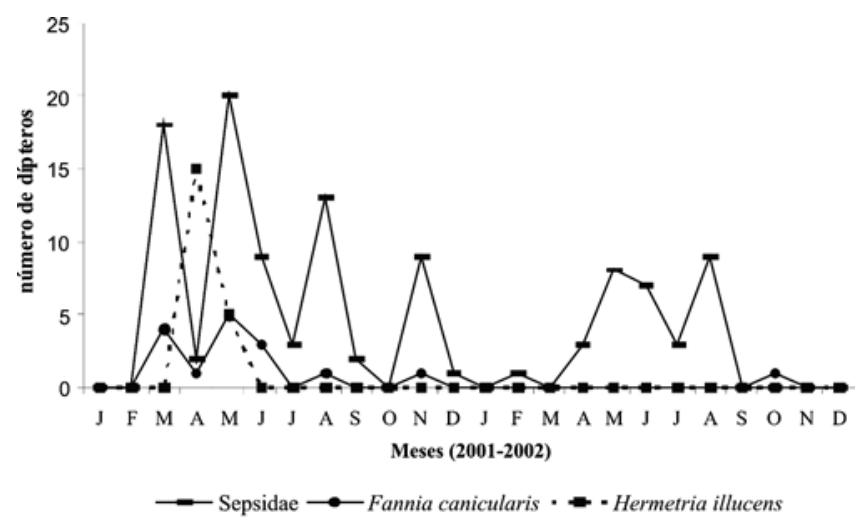

Figura 4. Dinâmica populacional de Sepsidae, Fannia canicularis e Hermetria illucens capturadas por armadilhas "jug-trap" durante os anos de 2001 e 2002, em granja avícola de São João da Boa Vista, São Paulo, Brasil. 
Tabela 2. Valores médios, desvios padrões, resultados das comparações múltiplas e análise de variância das diferentes espécies de dípteros capturados por armadilhas "jug-trap", durante os anos de 2001 e 2002 em granja avícola de São João da Boa Vista, São Paulo, Brasil.

\begin{tabular}{|c|c|c|c|c|c|c|c|c|}
\hline \multirow[t]{2}{*}{ Meses } & \multirow[t]{2}{*}{ Ano } & \multicolumn{7}{|c|}{ Espécies de moscas / Média e desvio padrão } \\
\hline & & $\begin{array}{c}\text { Drosophila } \\
\text { repleta }\end{array}$ & $\begin{array}{c}\text { Musca } \\
\text { domestica }\end{array}$ & Ophyra spp. & Sepsidae & $\begin{array}{c}\text { Hermetria } \\
\text { illucens }\end{array}$ & $\begin{array}{c}\text { Fannia } \\
\text { canicularis }\end{array}$ & $\begin{array}{c}\text { Chrysomya } \\
\text { meghacephala }\end{array}$ \\
\hline Janeiro & 2001 & $59927,5 \pm 0,1^{\mathrm{AB}}$ & $2041,0 \pm 0,3^{A}$ & $295,0 \pm 0,0^{A}$ & $0,0 \pm 0,0^{A}$ & $0,0 \pm 0,0^{A}$ & $0,0 \pm 0,7^{A}$ & $5,0 \pm 0,4^{A}$ \\
\hline Fevereiro & & $21432,0 \pm 0,5^{A}$ & $4332,5 \pm 0,2^{A}$ & $254,8 \pm 2,2^{\mathrm{AB}}$ & $0,0 \pm 0,0^{A}$ & $0,0 \pm 0,0^{A}$ & $0,0 \pm 0,0^{A}$ & $0,0 \pm 0,0^{B}$ \\
\hline Março & & $8218,6 \pm 0,2^{\mathrm{ABCD}}$ & $502,4 \pm 0,1^{\mathrm{A}}$ & $198,0 \pm 1,8^{\mathrm{BC}}$ & $3,6 \pm 0,3^{A}$ & $0,0 \pm 0,5^{\mathrm{A}}$ & $0,8 \pm 0,0^{A}$ & $0,0 \pm 0,0^{\mathrm{B}}$ \\
\hline Abril & & $5611,0 \pm 0,2^{A B C D}$ & $411,3 \pm 0,3^{\mathrm{AB}}$ & $168,3 \pm 1,2^{\mathrm{AB}}$ & $0,5 \pm 0,2^{A}$ & $3,8 \pm 0,2^{A}$ & $0,3 \pm 0,0^{A}$ & $0,0 \pm 0,0^{B}$ \\
\hline Maio & & $2116,4 \pm 0,2^{A B C D}$ & $326,2 \pm 0,2^{A B C}$ & $45,4 \pm 1,2^{\mathrm{BCD}}$ & $4,0 \pm 0,3^{\mathrm{A}}$ & $0,0 \pm 0,6^{A}$ & $1,0 \pm 0,0^{A}$ & $0,0 \pm 0,0^{B}$ \\
\hline Junho & & $68,3 \pm 0,3^{\mathrm{BCDEF}}$ & $70,3 \pm 0,4^{\mathrm{BCD}}$ & $18,5 \pm 0,4^{\mathrm{CD}}$ & $2,3 \pm 0,3^{A}$ & $0,0 \pm 0,5^{\mathrm{A}}$ & $0,8 \pm 0,0^{A}$ & $0,0 \pm 0,0^{B}$ \\
\hline Julho & & $37,0 \pm 0,2^{\mathrm{DEF}}$ & $19,3 \pm 0,4^{\mathrm{D}}$ & $2,3 \pm 0,5^{\mathrm{CD}}$ & $0,8 \pm 0,0^{A}$ & $0,0 \pm 0,3^{A}$ & $0,0 \pm 0,0^{A}$ & $0,0 \pm 0,0^{\mathrm{B}}$ \\
\hline Agosto & & $333,8 \pm 0,6^{\mathrm{CDEF}}$ & $84,3 \pm 0,5^{D}$ & $8,2 \pm 0,7^{\mathrm{BCD}}$ & $2,2 \pm 0,1^{A}$ & $0,0 \pm 0,3^{A}$ & $0,2 \pm 0,0^{A}$ & $0,0 \pm 0,0^{\mathrm{B}}$ \\
\hline Setembro & & $21,3 \pm 0,5^{\mathrm{ABCD}}$ & $355,7 \pm 0,7^{C D}$ & $12,0 \pm 0,6^{\mathrm{CD}}$ & $0,7 \pm 0,0^{A}$ & $0,0 \pm 0,3^{A}$ & $0,0 \pm 0,0^{A}$ & $0,0 \pm 0,0^{B}$ \\
\hline Outubro & & $13634,0 \pm 0,3^{A B C}$ & $1423,0 \pm 0,3^{\mathrm{CD}}$ & $6,8 \pm 1,8^{\mathrm{AB}}$ & $0,0 \pm 0,0^{A}$ & $0,0 \pm 0,0^{A}$ & $0,0 \pm 0,0^{A}$ & $0,0 \pm 0,0^{B}$ \\
\hline Novembro & & $27706,0 \pm 1,5^{\mathrm{ABC}}$ & $2255,0 \pm 0,7^{\mathrm{BCD}}$ & $31,6 \pm 2,0^{\mathrm{AB}}$ & $1,8 \pm 0,1^{\mathrm{A}}$ & $0,0 \pm 0,4^{\mathrm{A}}$ & $0,2 \pm 0,2^{\mathrm{A}}$ & $0,6 \pm 0,0^{B}$ \\
\hline Dezembro & & $39644,8 \pm 0,2^{\mathrm{A}}$ & $2890,8 \pm 0,1^{\mathrm{ABC}}$ & $86,8 \pm 0,1^{A}$ & $0,3 \pm 0,0^{A}$ & $0,0 \pm 0,2^{A}$ & $0,0 \pm 0,0^{A}$ & $0,0 \pm 0,0^{B}$ \\
\hline Janeiro & 2002 & $40323,5 \pm 0,1^{\mathrm{AB}}$ & $2136,5 \pm 0,2^{\mathrm{ABC}}$ & $93,0 \pm 0,0^{A}$ & $0,0 \pm 0,0^{A}$ & $0,0 \pm 0,0^{A}$ & $0,0 \pm 0,0^{A}$ & $0,0 \pm 0,0^{B}$ \\
\hline Fevereiro & & $39618,0 \pm 0,0^{A}$ & $4446,0 \pm 0,0^{A B}$ & $158,0 \pm 0,0^{A}$ & $1,0 \pm 0,0^{A}$ & $0,0 \pm 0,0^{A}$ & $0,0 \pm 0,0^{A}$ & $0,0 \pm 0,0^{B}$ \\
\hline Março & & $41646,5 \pm 0,1^{\mathrm{A}}$ & $2375,0 \pm 0,1^{A B C}$ & $85,5 \pm 0,0^{A}$ & $0,0 \pm 0,0^{A}$ & $0,0 \pm 0,0^{A}$ & $0,0 \pm 0,0^{A}$ & $0,0 \pm 0,0^{B}$ \\
\hline Abril & & $26861,5 \pm 0,2^{\mathrm{ABC}}$ & $1649,0 \pm 0,1^{\mathrm{ABC}}$ & $105,5 \pm 0,3^{A}$ & $1,5 \pm 0,0^{A}$ & $0,0 \pm 0,4^{\mathrm{A}}$ & $0,0 \pm 0,0^{A}$ & $0,0 \pm 0,0^{\mathrm{B}}$ \\
\hline Maio & & $4016,3 \pm 0,2^{\mathrm{ABCD}}$ & $466,3 \pm 0,4^{\mathrm{ABCD}}$ & $37,7 \pm 1,3^{\mathrm{BC}}$ & $2,7 \pm 0,0^{A}$ & $1,7 \pm 0,4^{\mathrm{A}}$ & $0,0 \pm 0,0^{A}$ & $0,0 \pm 0,4^{A B}$ \\
\hline Junho & & $82,0 \pm 0,4^{A B C D E F}$ & $227,5 \pm 0,1^{\mathrm{ABCD}}$ & $25,0 \pm 0,0^{B C D}$ & $3,5 \pm 0,0^{A}$ & $0,0 \pm 0,4^{A}$ & $0,0 \pm 0,0^{A}$ & $0,0 \pm 0,0^{B}$ \\
\hline Julho & & $14,0 \pm 0,0^{F}$ & $1,0 \pm 0,0^{\mathrm{D}}$ & $1,0 \pm 0,2^{\mathrm{CD}}$ & $1,5 \pm 0,0^{A}$ & $0,0 \pm 0,4^{A}$ & $0,0 \pm 0,0^{A}$ & $0,0 \pm 0,0^{B}$ \\
\hline Agosto & & $90,0 \pm 0,0^{\mathrm{EF}}$ & $2,0 \pm 0,1^{\mathrm{CD}}$ & $5,0 \pm 0,0^{\mathrm{BCD}}$ & $4,5 \pm 0,0^{A}$ & $0,0 \pm 0,2^{A}$ & $0,0 \pm 0,0^{A}$ & $0,0 \pm 0,0^{B}$ \\
\hline Setembro & & $3,0 \pm 0,0^{\mathrm{DEF}}$ & $10,0 \pm 0,2^{D}$ & $4,5 \pm 0,0^{\mathrm{D}}$ & $0,0 \pm 0,0^{A}$ & $0,0 \pm 0,0^{A}$ & $0,0 \pm 0,0^{A}$ & $0,0 \pm 0,0^{B}$ \\
\hline Outubro & & $1645,3 \pm 0,6^{\mathrm{EF}}$ & $7,3 \pm 0,0^{\mathrm{D}}$ & $2,0 \pm 0,0^{A B}$ & $0,0 \pm 0,2^{A}$ & $0,0 \pm 0,0^{A}$ & $0,3 \pm 0,0^{A}$ & $0,0 \pm 0,0^{B}$ \\
\hline Novembro & & $3261,0 \pm 0,1^{A B C D E}$ & $252,0 \pm 0,3^{C D}$ & $6,5 \pm 1,8^{\mathrm{BC}}$ & $0,0 \pm 0,0^{A}$ & $0,0 \pm 0,0^{A}$ & $0,0 \pm 0,2^{A}$ & $0,5 \pm 0,0^{B}$ \\
\hline Dezembro & & $7183,0 \pm 0,0^{\mathrm{ABCD}}$ & $520,0 \pm 0,1^{A B C D}$ & $34,0 \pm 0,0^{A B}$ & $0,0 \pm 0,0^{A}$ & $0,0 \pm 0,0^{A}$ & $0,0 \pm 0,0^{A}$ & $0,0 \pm 0,0^{B}$ \\
\hline Valor de F & & 9,21 & 10,53 & 2,97 & 0,40 & 1,21 & 1,87 & 3,79 \\
\hline Probabilidade & e sig. de & $\mathrm{F}<0,0001$ & $<0,0001$ & 0,0006 & 0,9902 & 0,2750 & 0,3013 & $<0,0001$ \\
\hline Coeficiente de & Variaçãc & io $\quad 22,36$ & 26,92 & 42,45 & 382,99 & 147,48 & 440,28 & 313,61 \\
\hline
\end{tabular}

${ }^{1}$ Médias seguidas por pelo menos uma letra em comum, na coluna, não diferem entre si pelo teste Tukey $(P \geq " 0,05)$.

Olton e Legner (1970) foram mais abundantes nos meses de inverno, quando os autores relataram que espécies deste gênero podem se constituir em uma praga mas que, normalmente, suas populações declinam rapidamente quando expostas a temperaturas mais elevadas. Essa variação de freqüência para $F$. canicularis em função da temperatura não foi observada neste trabalho, possivelmente em decorrência da baixa amostragem populacional constatada durante todo o período (Tabela 3).

Fannia canicularis foi encontrada por Bicho et al. (2004) em granja aviária de Pelotas, RS e por Bruno et al. (1993) em municípios de São Paulo, juntamente com F. trimaculata (Steim, 1898) e F. pusio (Wiedemann, 1830). Lomônaco e Prado (1994) em granja de aves poedeiras do município de Uberlândia, MG, também capturaram poucos fanídeos, dentre os quais, F. trimaculata e F. pusio.

Freqüentemente encontrado em aviários, o estratiomídeo $H$. illucens foi coletado em pequeno número (Tabela 1). Este fato pode estar relacionado com o longo período de desenvolvimento pupal ( \pm nove meses) deste inseto (BORROR; WHITE, 1970), uma vez que em São João da Bela Vista, as fezes foram retiradas do galpão de coleta a cada seis meses, aproximadamente.

Referente aos meses dos anos de 2001 e 2002, verifica-se que as análises de variância apresentaram resultados estatisticamente significativos $(\mathrm{P}<0,05)$ nos meses de coleta (Tabela 2) para: D. repleta, $M$. domestica, Ophyra spp. e $C$. megacephala e para os índices pluviométricos dentro de cada

Tabela 3. Coeficiente de correlação de Pearson das variáveis (temperatura, umidade e precipitação pluviométrica) para as diferentes espécies de dípteros capturados por armadiIhas "jug-trap", durante os anos de 2001 e 2002 em granja avícola de São João da Boa Vista, São Paulo, Brasil.

\begin{tabular}{lccc}
\hline Família/Gênero/Espécie & \multicolumn{3}{c}{ Coeficiente de correlação $(\mathrm{r})$} \\
\cline { 2 - 4 } & $\begin{array}{c}\text { Temperatura } \\
\left({ }^{\circ} \mathrm{C}\right)\end{array}$ & $\begin{array}{c}\text { Umidade } \\
(\%)\end{array}$ & $\begin{array}{c}\text { Precipitação } \\
\text { pluviométrica } \\
\left(\mathrm{mm}^{3}\right)\end{array}$ \\
\hline Drosophila repleta & $0,254 \mathrm{~ns}$ & $0,014 \mathrm{~ns}$ & $0,551^{*}$ \\
Musca domestica & $0,221 \mathrm{~ns}$ & $0,050 \mathrm{~ns}$ & $0,520^{*}$ \\
Ophyra spp. & $-0,113 \mathrm{~ns}$ & $0,211 \mathrm{~ns}$ & $-0,103 \mathrm{~ns}$ \\
Hermetria illucens & $-0,095 \mathrm{~ns}$ & $-0,231 \mathrm{~ns}$ & $-0,092 \mathrm{~ns}$ \\
Fannia canicularis & $-0,160 \mathrm{~ns}$ & $0,261 \mathrm{~ns}$ & $-0,055 \mathrm{~ns}$ \\
Sepsidae & $-0,054 \mathrm{~ns}$ & $0,058 \mathrm{~ns}$ & $-0,171 \mathrm{~ns}$ \\
Chrysomya megacephala & $-0,062 \mathrm{~ns}$ & $-0,002 \mathrm{~ns}$ & $-0,015 \mathrm{~ns}$ \\
\hline
\end{tabular}

* Significativo $(P<0,05)$ para índice pluvial;

** Significativo $(P<0,01)$ para índice pluvial;

${ }^{n s}$ Significativo $(P>0,05)$ para índice pluvial. 
família e/ou espécie: Drosophilidae (D. repleta) e Muscidae (M. domestica) (Tabela 3).

Já para Sepsidae, $H$. illucens e F. canicularis, não foram encontradas diferenças estatísticas significativas $(\mathrm{P}>0,05)$ entre meses de coleta, índices pluviais e demais covariáveis analisadas (Tabela 2 e 3). As correlações entre os totais de dípteros encontrados nas armadilhas e os dados climáticos para estas espécies (umidade relativa, temperatura e precipitação pluvial), não foram significativas (Tabela 3).

De modo geral, as variações no número de indivíduos de $D$. repleta e $M$. domestica ao longo dos anos, tiveram relações diretas com variações climáticas, principalmente precipitação pluvial $(\mathrm{P}<0,05)$, apresentando correlação positiva com esta covariável (Tabela 3) ou seja, o número de moscas aumentou significativamente nos meses em que houve aumento das chuvas. Assim, D. repleta e M. domestica apresentaram um único pico populacional ao longo de 12 meses. No mês de julho, tanto em 2001, quanto em 2002, constatou-se um baixo número dos dípteros supracitados (Figuras 1 e 2).

Bicho et al. (2004) em granja aviária de Pelotas, RS, registrou maior número de moscas (9.092) em outubro e julho. Já o menor índice de captura foi assinalado em abril (658), o que também coincidiu com a abrupta queda na temperatura mensal. Neste caso, $D$. repleta apresentou acme populacional em maio (1.695) e a menor captura deste díptero foi verificada no mês de março (136). Num estudo de artrópodes associados ao excremento de aves poedeiras, em Uberlândia, MG, Fernandes et al. (1995) notificaram um elevado índice na captura de uma espécie não identificada de Drosophilidae, em 10 dos 11 meses de amostragem, cujos picos populacionais foram constatados em fevereiro, março e agosto.

Em granjas de diversos locais dos Estados Unidos, as espécies mais comuns são M. domestica, Muscina stabulans (Fallén, 1817) e Stomoxys calcitrans (Linnaeus, 1758) (LEGNER et al., 1967; LEGNER; POORBAUGH, 1972; LEGNER et al., 1975ab; AXTELL; RUTZ, 1986; AXTELL; ARENDS, 1990).

De modo geral, o número de dípteros capturados é influenciado pela temperatura, com redução das populações nos meses com temperaturas amenas, uma vez que moscas dependem de temperaturas relativamente altas para se desenvolverem (MERCHANT et al., 1985; MENDES; LINHARES, 2002). Entretanto, segundo Pinto-Coelho (2000), além dos fatores climáticos, (temperatura, umidade relativa do ar e precipitação pluviométrica), outros fatores podem influenciar a sazonalidade dos artrópodes, como os dependentes da densidade (disponibilidade de alimento, espaço, predação) e os fatores independentes da densidade (componentes genéticos, interação social e dispersão).

Drosophila repleta parece estar assumindo importância crescente do ponto de vista epidemiológico nessa e em outras regiões (BICHO et al., 2004), uma vez que este díptero pode ser encontrado em granjas aviárias, e, em São João da Boa Vista, estado de São Paulo, correspondeu a cerca de $91 \%$ da população de moscas capturadas. Entretanto, convém salientar que este muscóide provavelmente não estava se desenvol- vendo no local, já que estágios imaturos dessa espécie não foram capturados no excremento das aves, por Lopes et al. (2007) que realizaram no mesmo local e período estudo com armadilhas apropriadas para larvas e pupas, durante os 24 meses de amostragem (LOPES et al., 2007).

Agradecimentos: A Dr ${ }^{\mathrm{a}}$ Marisa Rossi Monteiro pela colaboração técnica e aos professores Maria Cândida de Oliveira Costa, Afonso Celso Navarro e Dr. Gilson Pereira de Oliveira pelos comentários e sugestões.

\section{REFERÊNCIAS BIBLIOGRÁFICAS}

AVANCINI, R.M.P.; UETA, M.T. Manure breeding insects (Diptera and Coleoptera) responsable for cestoidosis in caged layer hens. Journal of Applied Entomology, v. 110, n. 3, p. 307-312, 1990.

AVANCINI, R.M.P.; SILVEIRA, G.A.R. Age structure in populations of muscoid flies from a poultry facility in Southeast Brazil. Memórias do Instituto Oswaldo Cruz, v. 95, n. 2, p. 259-264, 2000.

AXTELL, R.C. Fly management in poultry production cultural, biological and chemical. Poultry Science, v. 65, n. 4, p. 657-667, 1986.

AXTELL, R.C.; RUTZ, D.A. Role of parasites and predators as biological fly control agents in poultry production facilities. In. PATTERSON, R.S.; RUTZ, D.A. Biological control of muscoid flies. Entomological Society of American: Miscellaneous Publication, n. 61, p. 88-100, 1986.

AXTELL, R.C; ARENDS, J.J. Ecology and management of arthropods pest of poultry. Annual Review of Entomology, v. 35, n. 2, p. 101-126, 1990.

BANZATTO, D.A.; KRONCA, S.N. Experimentação Agrícola. Jaboticabal: FUNEP/FCAV/UNESP, 1989. 247p.

BICHO, C.L.; ALMEIDA, L.M.; RIBEIRO, P.B.; JÚNIOR, P.S. Flutuação de Diptera em granja avícola Pelotas, Rio Grande do Sul, Brasil. Iheringia: Série Zoológica. v. 94, n. 2, p. 205-210, 2004.

BORROR, J.D.; WHITE, E.R. Insects. Boston: Houghton Mifflin Company, 1970. 404p.

BRUNO, T.V.; GUIMARÃES, A.M.M; SANTOS, A.M.M.; TUCCI, E.C. Moscas sinantrópicas (Diptera) e seus predadores que se criam em esterco de aves poedeiras confinadas no Estado de São Paulo, Brasil. Revista Brasileira de Entomologia, v. 37, n. 3, p. 577-590, 1993.

BURG, J.G.; AXTELL, R.C. Monitoring house fly, Musca domestica (Diptera: Muscidae), population in caged-layer poultry houses using jug-trap. Environamental Entomology, v. 13, n. 2, p. 1083-1090, 1984.

CARRARO, M.V.; AZEVEDO, E.M.V.M. Controle alternativo contra pragas de interesse médico-veterinário e sanitário. Revista Brasileira de Medicina Veterinária, v. 22, n. 4, p. 22-29, 2000.

CARVALHO, C.J.B.; RIBEIRO, P.B. Chave de identificação das espécies de Calliphoridae (Diptera) do Sul do Brasil. 
Revista Brasileira de Parasitologia Veterinária, v. 19, n.2, p. 169-173, 2000.

CURRAN, C.H. The families and genera of North Americam Diptera. 2 ed. New York: Woodhaven, 1965. 515p.

FERNANDES, M.A.; SANTOS, M.A.S.; LOMÔNACO, C. Ocorrência de artrópodes no esterco acumulado em uma granja de galinhas poedeiras. Anais da Sociedade Entomológica do Brasil, v. 24, n. 3, p. 649-54, 1995.

GIANIZELLA, S.L. Coleópteros predadores de dípteros sinantrópicos (Coleoptera: Histeridae) em granja de aves poedeiras: levantamento, abundância, distribuição sazonal e interações com Musca domestica L., (Diptera: Muscidae) 1995. 36f. Dissertação (Mestrado) - Universidade Estadual de Campinas, Campinas, 1995.

GIANIZELLA, S.L.; PRADO, A.P. Levantamento e sazonalidade de coleópteros (Histeridae) em criação de aves poedeiras. Anais da Sociedade Entomológica Brasileira, v. 27, n. 4, p. 551-57, 1998.

GREENBERG, B. Flies and Disease - ecology, classification and biotic association. v. 1, New Jersey: University Press, 1971. 856p.

GUIMARÃES, J.H. Moscas sinantrópicas: perspectivas de manejo integrado em aviários no Estado de São Paulo. Agroquímica Ciba Geigy, v. 28, n. 1, p. 19-24, 1985.

HOFSTAD, M.S.; CALNEK, B.W.; HEMBOLT, C.T.; REID, W.M.; YODER, H.W. 1984. Diseases of Poultry. Ames: Iowa States Univ. Press, 1984. 71p.

KREBS, C.J. Ecological methods. New York: Haper and Row, xii, 1989. 700p.

LEGNER, E.F.; BAY, E.C.; WHITE, E.B. Activity of parasites from Diptera Musca domestica, Stomoxys calcitrans, Fannia caniculares and F. femoralis at sites in the Western Hemisphere. Annals of the Entomological Society of America, v. 60, n.2, p. 462-468, 1967.

LEGNER, E.F.; POORBAUGH, Jr. H. Biological control of vector and noxious synantropic flies: a review. California Vectors View, v. 11, n.11, p. 81-100, 1972.

LEGNER, E.F.; OLTON, G.S.; EASTWOOD, R.E.; DIETRICH, E.J. Seasonal density, distributions and interactions of predatory and scavenger arthropods in accumulation poultry wastes in coastal and interior Southern California. Entomophaga, v.3, n. 3, p. 269-283, 1975a.

LEGNER, E.F.; BOWEN, W. R.; ROONEY, W. F.; MCKEEN, W. D.; JOHNSTON, G. H. Integrated fly control on poultry ranches. California Agriculture. v. 29, n. 1, p. 8-10, 1975b.

LOMÔNACO, C.; PRADO, A.P. Estrutura comunitária e dinâmica populacional da fauna de dípteros e seus inimigos naturais em granjas avícolas. Anais da Sociedade Entomológica do Brasil, v. 23, n.1, p. 71-80, 1994.

LOPES, W.D.Z.; COSTA, F.H.; LOPES, W.C.Z.; BALIEIRO, J.C.C.; SOARES, V.E.; PRADO, A.P. Artrópodes associa- dos ao excremento de aves poedeiras. Neotropical Entomology. v. 36, n.4, p. 597-604, 2007.

LYSYK, T.J.; AXTELL, R.C. Movement and distribution of house flies (Diptera: Muscidae) between habitats in two livestock farms. Journal of Economic Entomology, v. 79, n. 4, p. 993-98, 1986.

MARICONI, F.A.; GUIMARÃES, J.H.; BERTI FILHO, E. A mosca doméstica e algumas outras moscas nocivas. Piracicaba: Fundação de Estudos Agrários Luiz de Queiroz, 1999. 135p.

MENDES, J.; LINHARES, A.X. Atratividade por iscas, sazonalidade e desenvolvimento ovariano em várias espécies de Muscidae (Diptera). Revista Brasileira de Entomologia, v.37, n. 2, p. 289-297, 1993.

MENDES, J.; LINHARES, A.X. Cattle Dung Breeding Diptera in Pasteur in Southeastern Brazil: Diversity, Abundance and Seasonality. Memórias do Instituto Oswaldo Cruz, v. 97, n. 1, p. 37-41, 2002.

MERCHANT, M.E.; FLANDERS, R.V.; WILLIANS, R.E. Sampling methods comparisons for estimation of parasitism of Musca domestica (Diptera: Muscidae) pupae in accumulated poultry manure. Journal of Economic Entomology, v. 78, n. 4, p. 1299-1303, 1985.

MILLER, B.F.; TEOTIA, J.S.; THATCHER, T.O. Digestion of poultry manure by Musca domestica. British Poultry Science, v. 15, n. 3, p. 231-4, 1988.

NORTH, M.O.; BELL, D.D. Commercial chicken production manual. 4 ed., New York: Van Nostrand Reinhold, 1990. 422p.

OLTON, G.S.; LEGNER, E.F. Winter inoculative realises of parasitoids to reduce houseflies in poultry manure. Journal of Economic Entomology, v. 68, n. 1, p. 35-38, 1970.

PECK, R.F.; ANDERSON, J.R. Arthropod predators of immature Diptera developing in poultry groupings in Northern California. Journal of Medical Entomology, v. 6, n.2, p. 163-7, 1969.

PECK, J.H.; ANDERSON, J.R. Influence of poultry manure removal schedules on various diptera larval and selected arthropod predators. Journal of Economic Entomology, v. 63, n. 1, p. 69-71, 1970.

POLVONY, D. Flies and diseases: ecology, classification and biotic association. Princeton: University Press, 1971. 83p.

PINTO-COELHO, R.M. Fundamentos de ecologia. Porto Alegre: Artmed, 2000. 247p.

SAS. User'S Guide: Basic and statistic. Cary: SAS. 1995. $1.686 \mathrm{p}$.

STAFFORD, K.C., BAY, D.E. Dispersion and association of house fly, Musca domestica (Diptera: Muscidae), larvae and both sexes of Macrocheles muscadomestica (Acari: Macrochelidae) in response to poultry manure moisture, temperature, and accumulation. Environmental Entomology, v. 16, n. 2, p. 159-64, 1987.

Recebido em 18 de janeiro de 2007.

Aceito para publicação em 20 de dezembro de 2007. 